\title{
Comportamiento de Conexión Losa-Columna en Nudos Interiores de Pórticos con Losas Planas y Vigas Embebidas
}

\author{
Bermeo Carla $^{1}$ iD $;$ Sánchez Diego ${ }^{1}$ * iD ; Placencia Patricio $^{1}$ (iD \\ ${ }^{1}$ Escuela Politécnica Nacional, Facultad de Ingeniería Civil y Ambiental, Quito, Ecuador
}

\begin{abstract}
Resumen: Las losas planas con vigas embebidas en estructuras de hormigón armado son ampliamente usadas en el Ecuador debido a su bajo costo, facilidad de construcción y aspecto arquitectónico. Las losas planas han demostrado que tienen un buen comportamiento ante cargas verticales, sin embargo, se han reportado numerosos colapsos estructurales en edificaciones con este sistema constructivo durante eventos sísmicos. El colapso producido en este tipo de estructuras es preocupante pues las fallas se han producido debido al punzonamiento de las losas alrededor de las columnas. Este tipo de falla ocurre de forma repentina y por lo tanto es necesario evitarla. No existe en el ACI la recomendación para el diseño de la conexión Losa - Columna, cuando se trata de pórticos sin muros o paredes estructurales. En este estudio, se construyeron dos modelos de prueba a escala real. Se construyeron 2 nudos interiores de un edificio con losas planas y fueron ensayadas ante una combinación de cargas verticales y laterales para observar el comportamiento de la conexión losa columna. La carga horizontal fue aplicada de forma cíclica y se colocó una sobre carga en la losa para simular la carga vertical de una edificación en servicio normal. Estos experimentos mostraron que el tipo de falla que se produce en estructuras de hormigón con losas planas es frágil. En ninguno de los dos modelos se produjo el agotamiento por flexión, sin embargo, sí se evidenciaron daños por corte en dos direcciones o punzonamiento.
\end{abstract}

Palabras clave: Conexión losa columna, hormigón armado, Diseño sismo resistente, punzonamiento.

\section{Experimental Test of Slab - Column Connection on Frames with Hidden Beams.}

\begin{abstract}
Flat slabs with hidden beams are widely used in reinforced concrete construction in Ecuador because of their low cost, short construction time and architectural appearance. Hidden beams are referred to beams that are as high as the flat slab. This kind of flat slabs has a good performance for vertical loads, however, several structural collapses have been reported during seismic events. These failures are commonly referred to as "punching shear" failures as the column appears to punch through the slab as the latter drops away from the failure surface around the column. These failures appear suddenly; therefore it is necessary to prevent them. In this experiment, two full scale specimens were constructed. Two interior slab - column connections were tested under gravity and seismic load to evaluate the slab column connection performance. The results showed that the failure of this structural system is fragile. In both specimens flexural yielding was not developed before the failure due to the punching shear.
\end{abstract}

Keywords: Seismic design, punching shear, reinforced concrete, slab-column connection.

\section{INTRODUCCIÓN}

En el Ecuador es muy común que se construyan edificios de hormigón armado con losas planas y vigas embebidas para edificaciones de pequeña y mediana altura. Este sistema estructural es usado en viviendas, edificios de oficinas, estacionamientos y centros comerciales.

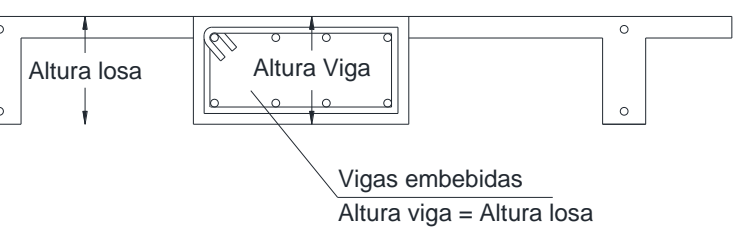

Figura 1. Losa plana alivianada con vigas embebidas.
Los edificios con losas planas y vigas embebidas son vulnerables al momento desbalanceado. El momento desbalanceado está presente en el nudo incluso cuando no existan fuerzas sísmicas. En este caso el momento desbalanceado se produce debido a la diferencia de longitud entre vanos contiguos, cargas diferentes a cada lado del nudo, diferencias de temperatura, etc. Cuando se produce un movimiento sísmico se incrementa el momento desbalanceado debido a las cargas laterales que afectan a la estructura. La combinación que se produce entre la carga vertical (peso propio y carga viva) y la carga horizontal debido al sismo causa que el punzonamiento sea asimétrico. Este efecto puede producir un daño severo a la estructura. 


\section{IMPORTANCIA DEL ESTUDIO}

En la Norma Ecuatoriana de la Construcción o NEC, está permitido el diseño y la construcción de edificios de hormigón armado con losas planas y vigas embebidas. El código NEC (2015) incluso otorga un factor de reducción de respuesta sísmica para edificios con losas planas y vigas embebidas. De acuerdo a la norma NEC (2015), el espesor mínimo de losa para edificios con losas planas alivianadas y vigas embebidas debe ser 250mm. El código NEC (2015) establece que los requisitos de diseño que se deben tomar en cuenta para edificios con losas planas y vigas embebidas son los mismos que se deben utilizar para edificios con vigas peraltadas. Adicionalmente a los requisitos para vigas peraltadas, el código recomienda que se realice un chequeo a punzonamiento en losas. Para realizar el chequeo el código NEC (2015) establece que se debe obtener un momento desbalanceado considerando la máxima capacidad a flexión en ambos lados del nudo, esta es una de las recomendaciones de la referencia 6

El Ecuador está localizado es una zona de alto peligro sísmico. Esto se evidenció recientemente. El 16 de abril de 2016 a las 18:58:37 se produjo un sismo de magnitud $7.8 \mathrm{Mw}$ en Pedernales. Luego del evento sísmico se pudo evidenciar que varios edificios con losas planas y vigas embebidas llegaron al colapso, la mayoría de estos edificios no se diseñaron tomando en cuenta las recomendaciones del código de construcción vigente en Ecuador NEC 2015.

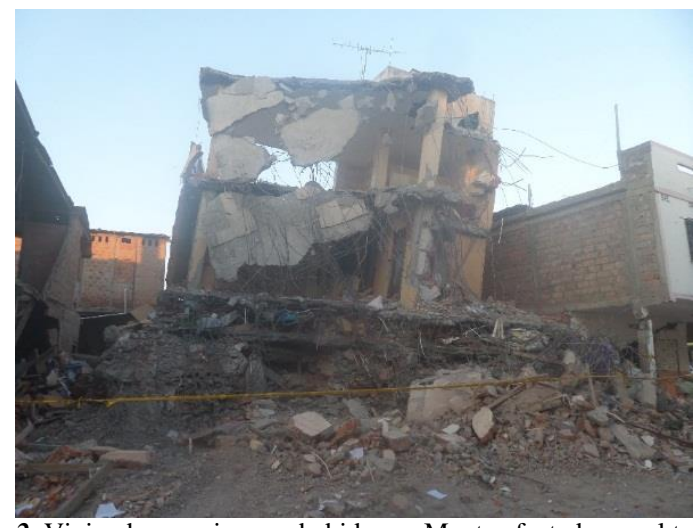

Figura 2. Vivienda con vigas embebidas en Manta afectada por el terremoto del 16 de abril de 2017.

Por lo expuesto anteriormente es importante realizar estudios experimentales para determinar el comportamiento de la conexión losa columna que se han diseñado y construido usando las recomendaciones de la Norma Ecuatoriana de la Construcción.

\section{DESCRIPCIÓN DEL ENSAYO}

\subsection{Edificio tipo}

Para llegar a determinar conclusiones y recomendaciones relevantes respecto al sistema estructural de losas planas con vigas embebidas será necesario realizar una gran cantidad de ensayos considerando variables como altura de losa, condiciones de carga vertical, refuerzo usado en la zona de punzonamiento, etc. Sin embargo, para una fase inicial, en este estudio se han considerado dos modelos representan un nudo interior de un edificio de 5 pisos. El edificio tipo es un edificio de hormigón armado con losas planas alivianadas y que contienen vigas embebidas que actúan como el sistema de soporte de cargas verticales. Las columnas serán las encargadas de resistir las fuerzas horizontales. El espesor de la losa alivianada es de $250 \mathrm{~mm}$, al tener vigas embebidas, la altura de las mismas es también de $250 \mathrm{~mm}$. Existen cuatro vanos en cada dirección de $4000 \mathrm{~mm}$ de longitud. La altura de entrepiso es de $2700 \mathrm{~mm}$. Las columnas son cuadradas de 400mm de lado. La carga muerta de servicio es de 6 962,0Pa. La carga viva de servicio es de $2451,0 \mathrm{~Pa}$. La carga horizontal aplicada se obtuvo del espectro de diseño en aceleraciones que se establece en el código NEC.

\subsection{Descripción del espécimen}

Se construyeron dos modelos a escala real. La longitud de losa en las dos direcciones es de $4000 \mathrm{~mm}$, la altura total de la columna es de $2700 \mathrm{~mm}$. La longitud de losa y de columna se construyó considerando que los puntos de inflexión ante carga lateral se encuentran en la mitad del vano y en la mitad de la altura de entrepiso. La distancia de centro a centro en los nervios de la losa es de 500mm.

Para obtener la cantidad de refuerzo longitudinal, en el primer ensayo, se realizó el análisis estructural del edificio tipo. Para el cálculo del acero transversal, se utilizaron las recomendaciones contenidas en la referencia 6. Según el cálculo realizado, cada rama de estribos debe tener una sección de $0.23 \mathrm{~cm} 2$. La cantidad de refuerzo transversal colocada es de $0.79 \mathrm{~cm} 2$.

En cuanto al segundo ensayo, por fines didácticos, se incrementó la cantidad de acero longitudinal. Esto debido a que, al aumentar la cantidad de acero longitudinal, se incrementa el problema de punzonamiento por momento desbalanceado. Al cambiar la cantidad de acero longitudinal, se hizo un recalculo de la cantidad de acero transversal. En este caso, según las recomendaciones de la referencia 6 , se necesita que cada rama tenga una sección de $1.04 \mathrm{~cm} 2$. La cantidad de acero colocada es de $0.79 \mathrm{~cm} 2$.

El refuerzo usado en el primer ensayo consistió en colocar en las vigas embebidas un área de acero longitudinal de: $\mathrm{As}_{\text {sup }}=15.27 \mathrm{~cm} 2$ y $\mathrm{As}_{\mathrm{inf}}=9.24 \mathrm{~cm} 2$ con $1 \mathrm{E} \emptyset 10 \mathrm{~mm} @ 5 \mathrm{~cm}$, según especificaciones de la Norma Ecuatoriana de la Construcción (NEC-15). Para el segundo ensayo se colocó en las vigas embebidas un área de acero longitudinal de: $\mathrm{As}_{\text {sup }}=$ $25.13 \mathrm{~cm} 2$ y $\mathrm{As}_{\mathrm{inf}}=16.08 \mathrm{~cm} 2 \mathrm{con} 1 \mathrm{E} \emptyset 10 \mathrm{~mm} @ 10 \mathrm{~cm}$.

El refuerzo utilizado tiene un esfuerzo mínimo de fluencia de 462,51MPa y un esfuerzo máximo de tensión de 607,14MPa. La resistencia del hormigón para el primer ensayo es de 24MPa. La resistencia del hormigón del Segundo ensayo es de $18 \mathrm{MPa}$. Adicional al peso propio de los modelos, se colocó una carga adicional de $3825 \mathrm{~N}$ sobre la losa. Esta carga se colocó para considerar la carga vertical normal que un edificio de oficinas podría tener antes de que se produzca un movimiento sísmico. Los efectos producidos por un evento sísmico se produjeron por dos gatos hidráulicos colocados en los bordes de las vigas embebidas en la dirección de análisis (ver Figura 3) 


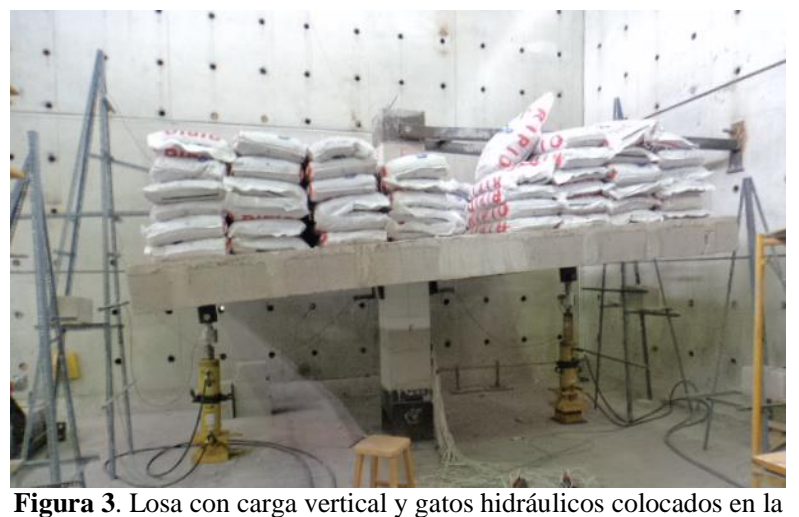

Figura 3. Losa con carga vertical y gatos hidráulicos colocados en la dirección de análisis.

\subsection{Instrumentación}

Para el primer ensayo se colocaron 40 Strain gauge para obtener la deformación unitaria del acero de refuerzo, su ubicación. Se colocaron dos LVDT en los bordes sobre las vigas embebidas.

Para el segundo ensayo se colocaron 38 Strain. Se colocaron dos LVDT en los bordes sobre las vigas embebidas.

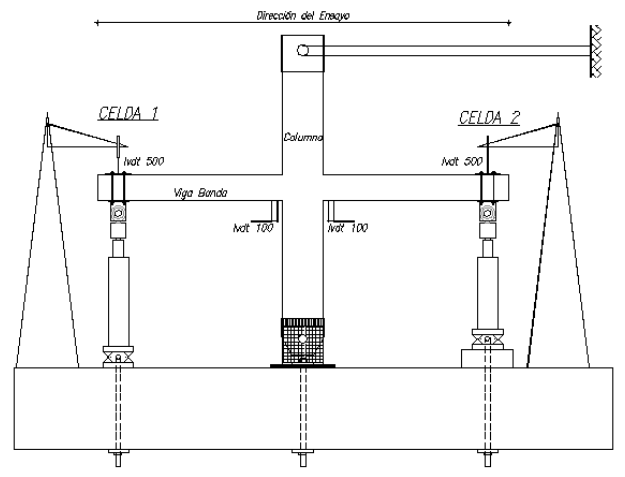

Figura 4. Esquema de instrumentos para el ensayo de la conexión losacolumna.

\section{RESULTADOS ESPERADOS}

\subsection{Curva momento rotación}

La curva momento rotación de la sección se obtuvo usando el software con licencia académica denominado "Seismostruct". La curva momento rotación del primer y segundo ensayo se muestran en la figura 5 .

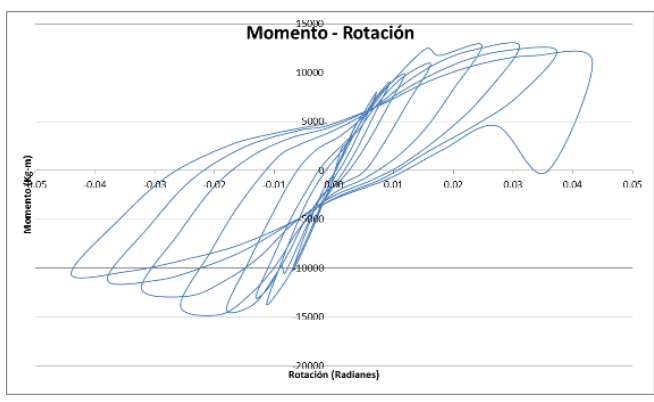

(A)

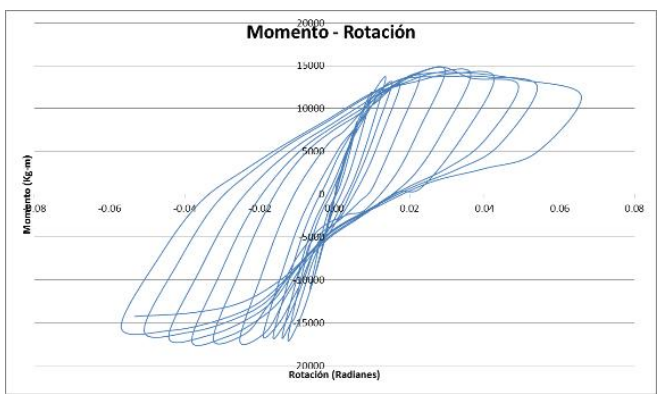

(B)

Figura 5. Curvas de momento-rotación esperadas según "Seismostruct" A) Prototipo 1, B) prototipo 2.

\subsection{Momento de fluencia y momento máximo}

Los momentos de fluencia y máximo se obtuvieron mediante la ecuación (1).

$$
M p=A s * \alpha * f y *\left(d-\frac{A s^{*} \alpha^{*} f y}{1,7 * f^{\prime} c^{*} b}\right)
$$

Donde $M p$ es momento probable (T-m), As es el refuerzo longitudinal ( $\mathrm{cm} 2), \alpha$ es el factor de sobre resistencia del acero ( $\sin$ unidades), $f y$ es el esfuerzo de fluencia $(\mathrm{Kg} / \mathrm{cm} 2), d$ es la distancia de la fibra extrema en compresión al centroide del refuerzo de acero en tensión (cm), $b$ ancho de compresión (cm), f'c resistencia de compresión del hormigón $(\mathrm{Kg} / \mathrm{cm} 2)$.

Para obtener el momento de fluencia, se aplica la fórmula (1) con un valor $\alpha=1,0$. Para obtener el momento máximo se aplica la fórmula (1) con un valor $\alpha=1,3$.

En la siguiente tabla se da a conocer los valores de los momentos que se espera de los prototipos.

Tabla 1. Resultados de momentos máximos y de fluencia de los prototipos.

\begin{tabular}{ccccc}
\hline \multirow{2}{*}{$\begin{array}{c}\text { DESCRIPCI } \\
\text { ÓN }\end{array}$} & $\begin{array}{c}c \\
\text { Poda la } \\
\text { losa }\end{array}$ & $\begin{array}{c}\text { Franja de } \\
\text { columna }\end{array}$ & Toda la losa & $\begin{array}{c}\text { Franja de } \\
\text { columna }\end{array}$ \\
\hline $\begin{array}{c}\text { Momento de } \\
\text { Fluencia (-) } \\
\text { Momento de }\end{array}$ & 16440 & 12480.8 & 22231,3 & 17730,0 \\
$\begin{array}{c}\text { Fluencia (+) } \\
\text { Momento de } \\
\text { resistencia } \\
\text { última (-) }\end{array}$ & 11140 & 8015,92 & 15738,5 & $13,410,0$ \\
$\begin{array}{c}\text { Momento de } \\
\text { resistencia } \\
\text { última (+) }\end{array}$ & 14400 & 10255,0 & 19743,5 & 16930,0 \\
\hline
\end{tabular}




\subsection{Ductilidad}

La ductilidad del sistema se obtiene con la ecuación (2) (Park y Paulay, 1973).

$$
u=\frac{\phi_{u}}{\phi_{y}}
$$

Donde u es la ductilidad del espécimen, $\phi_{u}$ es la curvatura para el momento máximo, $\phi_{y}$ es la curvatura para el momento de fluencia.

La ductilidad teórica obtenida para el ensayo 1 y 2 es 10,0 y 13,50 respectivamente. Este valor se obtuvo del diagrama momento curvatura que proporciona el programa Etabs, se presenta en la Figura (6)

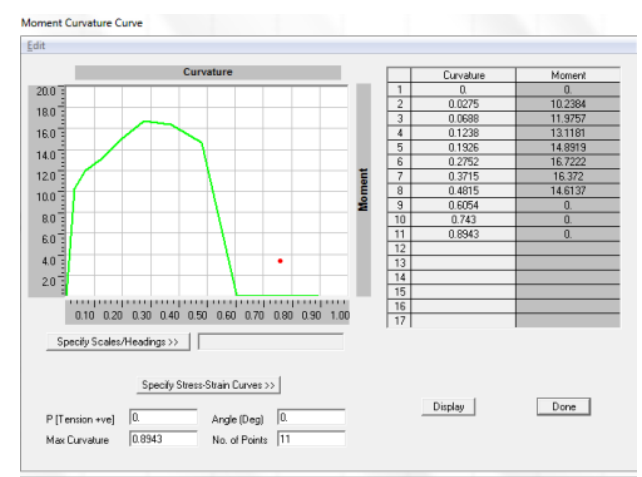

(a)

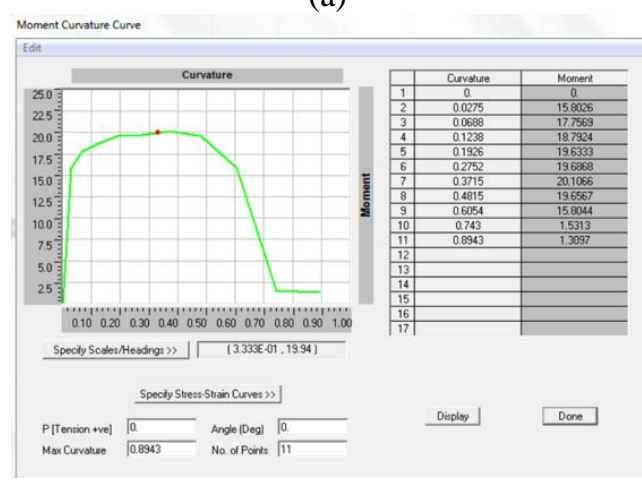

(b)

Figura 6. Diagrama momento-curvatura, a) prototipo 1, b) prototipo 2.

Debido a que la ductilidad de curvatura teórica se obtuvo del diagrama momento curvatura, este valor se debe dividir para cuatro (Park y Paulay, 1973), de esta forma se podrá comparar con la ductilidad de desplazamiento experimental obtenida del diagrama momento rotación.

Para la comparación con los resultados experimentales se usará una ductilidad de 2,5 para el primer ensayo y 3,38 para el segundo ensayo.

\section{RESULTADOS OBTENIDOS}

\subsection{Diagrama momento rotación}

A continuación se presenta el diagrama momento rotación experimental y se lo compara con el diagrama teórico (Figura 7).

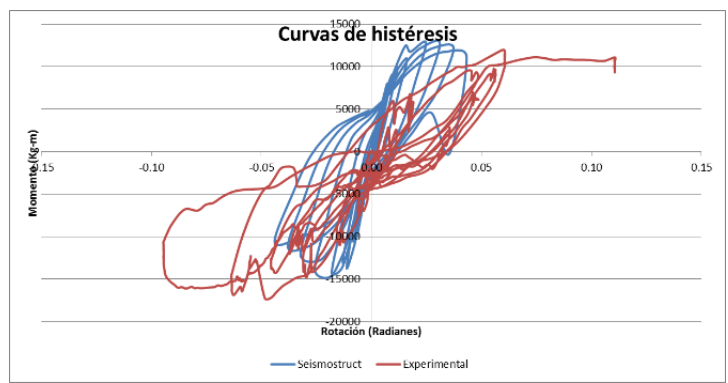

(a)

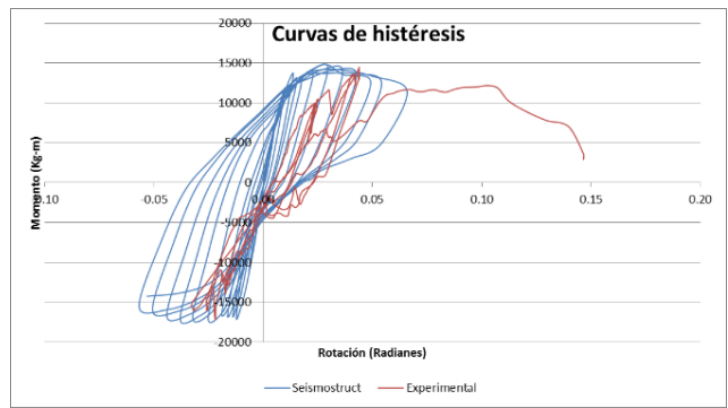

(b)

Figura 7. Comparación de curvas Momento-Rotación entre el teórico y experimental, a) prototipo 1, b) prototipo 2 .

\subsection{Momentos máximo y de fluencia}

Los momentos máximos y de fluencia de obtienen de la envolvente de la curva momento rotación. Las siguientes tablas indican sus resultados y diferencias.

\begin{tabular}{|c|c|c|c|c|}
\hline \multirow{2}{*}{$\begin{array}{c}\text { DESCRIPCI } \\
\text { ÓN }\end{array}$} & \multicolumn{2}{|c|}{ PROTOTIPO I } & \multicolumn{2}{|c|}{ PROTOTIPO II } \\
\hline & CELDA 1 & CELDA 2 & CELDA 1 & CELDA 2 \\
\hline $\begin{array}{l}\text { Momento de } \\
\text { Fluencia (-) }\end{array}$ & 10124,96 & 14414,3 & 17112,76 & 17184,6 \\
\hline $\begin{array}{l}\text { Momento de } \\
\text { Fluencia (+) }\end{array}$ & 8113,67 & 7266,4 & 10831,0 & 11205,3 \\
\hline $\begin{array}{c}\text { Momento de } \\
\text { resistencia } \\
\text { última (-) }\end{array}$ & 16315,2 & 16037,3 & & 19347,9 \\
\hline $\begin{array}{l}\text { Momento de } \\
\text { resistencia } \\
\text { última (+) }\end{array}$ & 12884,88 & 10986,9 & 14485,42 & \\
\hline
\end{tabular}

\subsection{Ductilidad}

Fue obtenida usando la ecuación (2).

La ductilidad para el primer ensayo fue de 2,7.

La ductilidad para el segundo ensayo fue de 1,75.

\subsection{Amortiguamiento viscoso equivalente}

Para obtener el amortiguamiento viscoso equivalente es necesario obtener la energía disipada, energía elástica y el amortiguamiento equivalente. Estos parámetros están en función de las fuerzas y desplazamientos de fluencia y últimas (Aguiar, 2012).

De acuerdo a los resultados obtenidos de las tablas, los valores escogidos para este cálculo, se obtienen de la envolvente 
fuerza desplazamiento de la celda 2 que se muestra en la Figura (8).

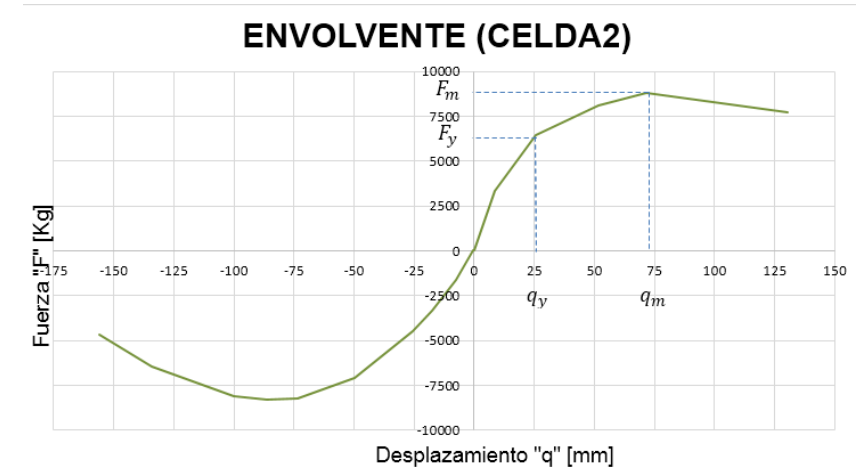

(a)

Fuerza-Desplazamiento Celda 2

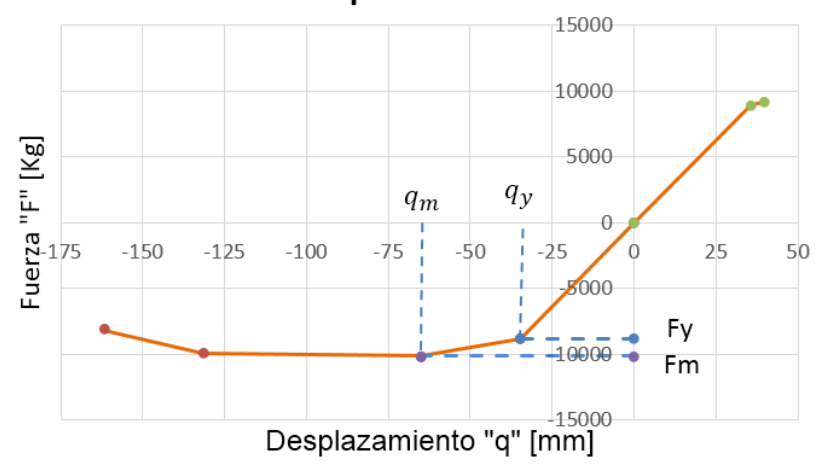

(b)

Figura 8. Envolvente de Fuerza-Desplazamiento, a) prototipo1, b) prototipo2.

La energía disipada se obtiene de la ecuación (3) (Aguiar, 2012).

$$
E_{D}=4 *\left(F_{y} * q_{u}-F_{u} * q_{y}\right)
$$

Donde $E d$ es la energía disipada (Kg-mm), $F y$ es la fuerza de fluencia $(\mathrm{Kg}), q u$ es el desplazamiento último $(\mathrm{mm}), F u$ es la carga máxima aplicada en el ciclo de histéresis $(\mathrm{Kg})$ y $q y$ es el desplazamiento en el punto de fluencia $(\mathrm{mm})$.

La energía disipada para el primer ensayo es $960123.81 \mathrm{Kg}$ $\mathrm{mm}$.

La energía disipada para el segundo ensayo es $875573.585 \mathrm{Kg}$ $\mathrm{mm}$.

La energía elástica está dada por la ecuación (4) (Aguiar, 2012).

$$
E s=\frac{F_{u} q_{u}}{2}
$$

Aplicando la ecuación, la energía elástica para cada prototipo, los resultados son para el primer ensayo Es es 315740.196 Kg.mm y para el segundo ensayo Es es 329316,815 Kg.mm El amortiguamiento equivalente viscoso, está dado por la siguiente ecuación (5) (Aguiar, 2012).

$$
\zeta_{e q}==\frac{E_{D}}{4 \pi E_{S}}
$$

El amortiguamiento equivalente $\zeta_{e q}$ del primer ensayo es 0.21 y del segundo ensayo es 0.19

\subsection{Agotamiento del sistema}

A continuación, se muestran fotografías que se tomaron luego de la falla del modelo. El área de punzonamiento de la unión losa-columna se refleja en la siguiente figura.

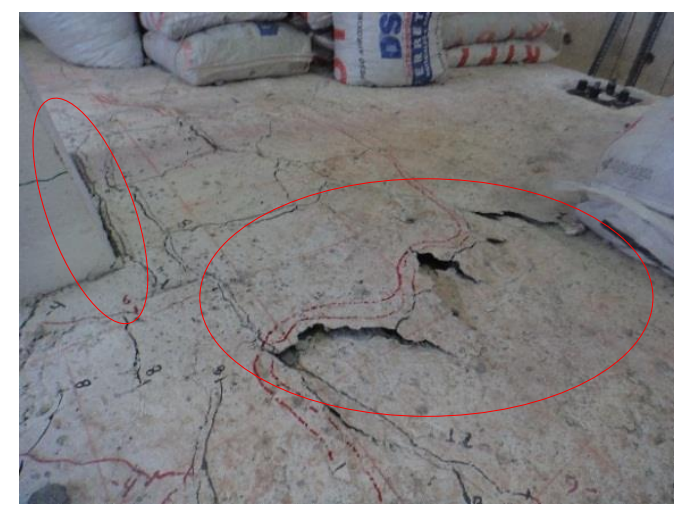

(a)

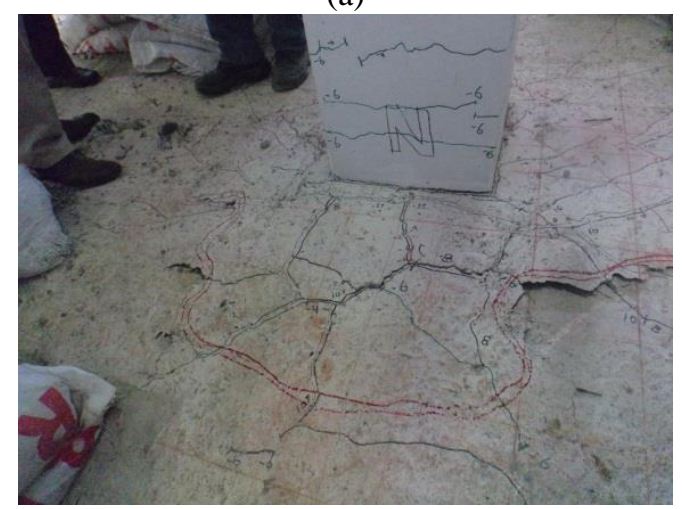

(b)

Figura 9. Zona de agotamiento, falla por punzonamiento. (a) Área de punzonamiento, (b)Fisura alrededor de columna.

El agotamiento produce un asentamiento en la losa debido al movimiento producido por las fuerzas proporcionadas de los gatos hidráulicos 


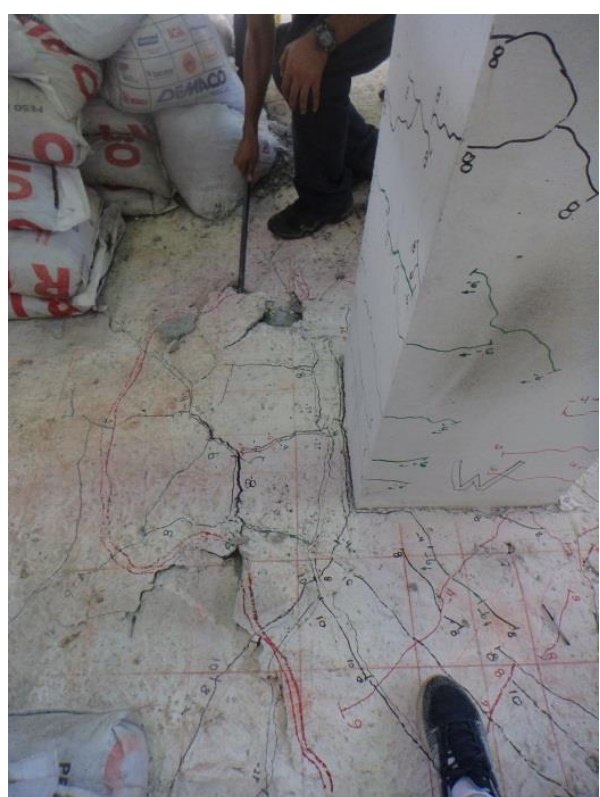

Figura 10. Losa agotada alrededor de la columna.

El agotamiento de la estructura por punzonamiento, produce el desprendimiento de la losa con respecto a la columna en forma cónica desde la unión inferior de la unión losa-columna hacia la zona superior de la losa a una distancia no mayor al peralte de la viga $(\mathrm{d})$.

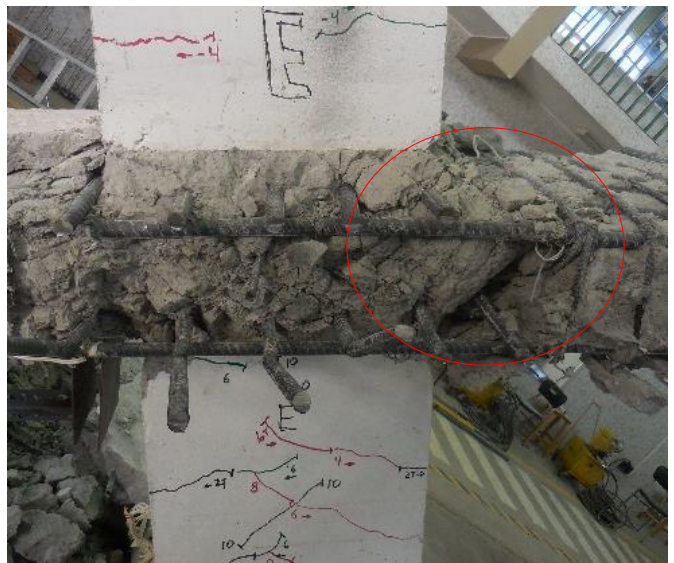

Figura 11. Efecto del corte de la losa debido a la falla por punzonamiento.

\section{CONCLUSIONES}

Se construyeron dos prototipos a escala real de un nudo interior de una estructura compuesta por losas planas. El primer ensayo consistió en colocar en las vigas embebidas un área de acero de: $\mathrm{As}_{\mathrm{sup}}=10.16 \mathrm{~cm} 2$ y $\mathrm{As}_{\mathrm{inf}}=6.16 \mathrm{~cm} 2$ con 1Eø10mm@5cm, según especificaciones de la Norma Ecuatoriana de la Construcción (NEC-15).

El segundo ensayo consistió en colocar en las vigas embebidas un área de acero de: $A s_{\text {sup }}=18.84 \mathrm{~cm} 2$ y As $s_{\text {inf }}=12.06 \mathrm{~cm} 2$ con 1Eø10mm@10cm, según criterios de cálculo del folleto "Diseños de edificios con vigas banda" por el Ing. Patricio Placencia. Cabe señalar que en el Ecuador, las vigas embebidas se conocen como vigas banda.

De los resultados se puede concluir:
La ductilidad de desplazamiento $\mu=2.7$, obtenida en el primer ensayo es similar a la ductilidad de desplazamiento $\mu=2.5$, que se calculó de forma teórica.

La ductilidad de desplazamiento $\mu=1.75$, obtenida en el segundo ensayo es aproximadamente la mitad de la ductilidad de desplazamiento $\mu=3.4$, que se calculó de forma teórica.

La diferencia entre la ductilidad de desplazamiento teórica y la experimental obtenida en el segundo ensayo se debe a que el prototipo tuvo una falla por punzonamiento poco después de que se inicie la fluencia en las varillas de refuerzo.

La ductilidad de desplazamiento obtenida en los dos prototipos está en un rango inferior al que se considera como mínimo para diseños sismo resistente $(\mu \geq 4.0)$.

En el primer ensayo el momento de fluencia y el momento máximo teóricos están muy cerca a los obtenidos de forma práctica.

En el prototipo 2 los momentos de fluencia obtenidos de forma teórica son similares a los momentos de fluencia obtenidos de forma experimental.

En el prototipo 1, se realizó en chequeo de la losa a punzonamiento ante carga vertical y momento desbalanceado provocado por la acción sísmica, este chequeo permitió que el prototipo presente resultados (ductilidad y resistencia) muy similares a los que se calculó de forma teórica.

La falla por punzonamiento en la losa se produjo de forma repentina.

En el segundo ensayo se tomó la medida del cono de la falla por punzonamiento en el nivel superior de la losa, el valor medido fue $14 \mathrm{~cm}$, esto corresponde aproximadamente a $2 \mathrm{~d} / 3$.

El amortiguamiento en el rango elástico de los prototipos 1 y 2 es de 0.16 .

El amortiguamiento de los prototipos 1 y 2 aumenta un poco cuando se encuentran trabajando en el rango inelástico, a un valor de 0.18 y 0.175 respectivamente.

Los prototipos ensayados pueden clasificarse como estructura tipo B de acuerdo a la clasificación determinada por el ATC40, se considera a este tipo de estructuras en el rango intermedio para un buen desempeño ante solicitaciones sísmicas.

Las losas planas o losas construidas con vigas embebidas son buenas ante carga vertical y no tienen buen comportamiento ante carga lateral debido a que no son suficientemente dúctiles.

La norma NEC-15 proporciona un valor para coeficiente de reducción sísmica $(\mathrm{R})$ igual a 5 para edificios con vigas embebidas, pero no proporcionan un método de diseño de la unión losa-columna que es la zona donde se presentan mayores esfuerzos.

La investigación actual se realizó en base al artículo "Diseño sismorresistente de edificios con losas con vigas banda (embebidas)" presentado en las "XI Jornadas de Ingeniería 
Estructural" que tuvieron lugar en la Universidad Santiago de Guayaquil en el año 1997, realizado por parte del Ing. Patricio Placencia.

Según las recomendaciones de este artículo, el primer ensayo cumplía con los requisitos de diseño a punzonamiento, el resultado mostró que los valores de resistencia a momento y ductilidad están cercanas a las obtenidas de forma teórica. No se evidenció falla por punzonamiento. El modelo tampoco se agotó en flexión. El ensayo tuvo que detenerse debido a problemas con las celdas de carga. El desplazamiento de la celda de carga no fue suficiente para agotar el sistema. Sin embargo, si se pudo apreciar fisuras alrededor de la zona de columna. Este es un signo de que se puede presentar una falla a punzonamiento.

En el segundo modelo, el diseño no era aceptable, se produjo falla por punzonamiento y no se obtuvieron valores de resistencia ni ductilidad cercanos a los teóricos. Por este motivo se recomienda que en el caso de que diseñe edificios con vigas embebidas, se realice el chequeo a punzonamiento usando la teoría que se presenta en la referencia 6.

Con este estudio se pretende iniciar un proceso investigativo de construcciones de edificios con vigas embebidas y verificar su comportamiento en zonas de alto peligro sísmico. Además es importante determinar si es posible mejorar el comportamiento del sistema para que pueda ser utilizado de forma segura.

\section{AGRADECIMIENTO}

Se agradece de manera especial al Ing. Patricio Placencia por haber apoyado y colaborado con sus conocimientos a esta investigación, así mismo un agradecimiento para quienes forman parte del Centro de Investigación de la Vivienda de la Escuela Politécnica Nacional, por su colaboración en las diferentes actividades antes y durante el ensayo de los prototipos.

\section{REFERENCIAS}

ACI318-14. (2015). Requisitos de Reglamento para Concreto Estructural (ACI 318-14). Farmington Hills, USA: IHS.

AGUIAR, R. (2012). Dinámica de Estructuras con CEINCI-LAB. Quito: Centro de Investigaciones Científicas. Escuela Politécnica del Ejército.

Cuevas., O. M. (2005). Apectos fundamentales del concreto reforzado. México: Limusa.

NEC. (2015). Peligro Sísmico, Diseño Sismoresistente (NEC_SE_DS). Quito: Dirección de Comunicación Social, MIDUVI.

NEC. (2015). Hormigón armado (NEC_SE_HM). Quito: Dirección de Comunicación Social, MIDUVI.

PATRICIO PLACENCIA, (1997). "Diseño Sismorresistente De Edificios Con Losas Con Vigas Banda". XI Jornadas de Ingeniería Estructural. Universidad Católica Santiago de Guayaquil.
Park, R. y Paulay, M. (1973) Reinforced Concrete Structures. New York.

\section{BIOGRAFÍAS}

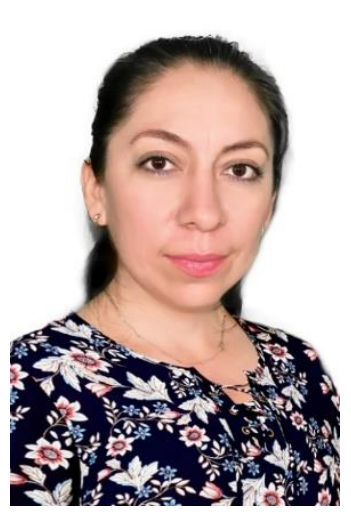

Carla Bermeo Fárez, Ingeniera Civil, Universidad Católica de Cuenca. Master en Estructuras, Escuela Politécnica Nacional, Quito. Profesora de Bachillerato del Colegio Nacional Camilo Gallegos Toledo. Profesora Titular de Pregrado de la carrera de Arquitectura de la Universidad Católica de Cuenca desde el 2016 hasta 2019. Consultora en el departamento de planificación del Gobierno

Autónomo Descentralizado de Gualaquiza. Consultora estructural de la empresa Astec Cia. Ltda.

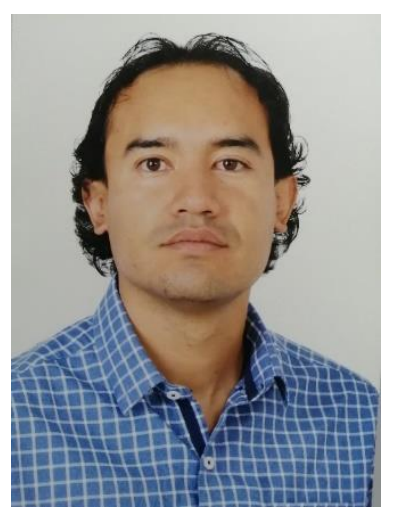

Diego Sánchez Oñate, Ingeniero Civil, Universidad Central del Ecuador. Magister en Estructuras, Escuela Politécnica Nacional. Profesor en la Universidad Central del Ecuador desde 2018. Consultor estructural para el diseño de 30 edificios públicos y privados en el Ecuador entre los que se encuentran hospitales, iglesias, centros comerciales, conjuntos habitacionales, escuelas y colegios. Consultor estructural para el reforzamiento de estructuras en la ciudad de Quito. Constructor de proyectos públicos y privados. 


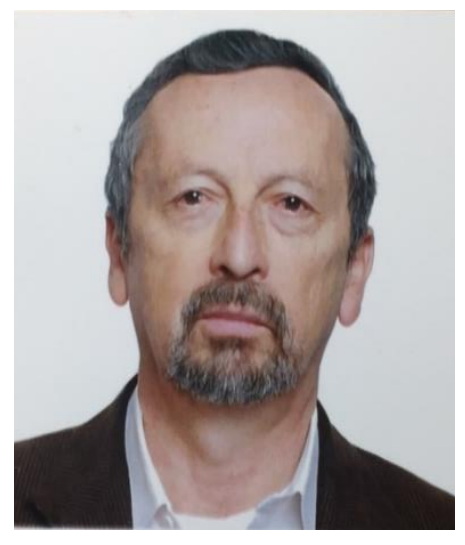

Patricio Placencia Andrade, Ingeniero Civil, Escuela Politécnica Nacional. Master of Science in Civil Engineering, U. Texas, en Austin, Estados Unidos. Fue Profesor Principal en la Escuela Politécnica Nacional, Quito, de cursos de pregrado y postgrado, desde 1979 hasta su jubilación en el 2014. Desde 2014 hasta la fecha sigue dando cursos como Profesor Honorario contratado. Formuló el proyecto de creación del Laboratorio de estructuras "Centro de Investigación de la Vivienda", siendo su Director hasta 2014. Impulsó y coordinó la creación del Postgrado de Ingeniería Estructural en la EPN. Ha sido el autor de artículos técnicos urgentes en su momento, como Metodología para estudio estructural de monumentos históricos, Diseño sismo resistente de edificios aporticados, Diseño sismo resistente de edificios con vigas banda, Diseño sismo resistente de edificios con muros, Vulnerabilidad sísmica en el Ecuador - mejores sistemas constructivos, Estructuras con mampostería enchapada. Ha escrito otros artículos técnicos de interés científico. Ha sido consultor estructural para el diseño estructural de más de 100 estructuras nuevas. Ha sido consultor estructural para el diagnóstico de la seguridad sísmica y su diseño del reforzamiento de más de 100 edificaciones existentes, entre edificios nuevos, antiguos, iglesias, casas, etc. Es consultor estructural de la OPS, PNUD, BID, Banco Mundial. Fue miembro del comité ACI 318-L, que elabora el Código ACI, desde 2005 hasta 2015. 